\title{
Les héros anonymes de la Révolution Mexicaine sur papier glacé: le juan et la soldadera comme symboles de "mexicanité"
}

\author{
Marion Gautreau*
}

\begin{abstract}
Résumé:
Au même titre que le muralisme des années vingt ou que la novela de la Revolución, les photographies de la guerre civile mexicaine de 1910 à 1920 ont contribué à la consolidation de l'idéologie post-révolutionnaire en offrant au lectorat des revues illustrées une représentation lissée et stéréotypée du conflit. Cet article se cantonne à l'analyse des images de juanes et de soldaderas publiées dans trois magazines de la Post-Révolution (1910-1920) afin de démontrer comment ces photographies enferment les acteurs de la guerre dans des "types" et les érigent en symboles de "mexicanité", relayant ainsi les discours simplificateurs des dirigeants post-révolutionnaires et gommant la complexité du conflit fondateur du Mexique du XXe siècle.
\end{abstract}

Mots-clefs: juan, soldadera, Révolution Mexicaine, photographie, presse illustrée, mexicanité.

Les photographies des juanes ${ }^{1}$ et des soldaderas ${ }^{2}$ de la Révolution Mexicaine circulent encore régulièrement aujourd'hui lorsqu'il s'agit d'évoquer la guerre civile du début du XXe siècle. Cependant, cet article se penche sur l’immédiat après-guerre, période communément appelée la Post-Révolution, dont la délimitation chronologique est controversée mais que nous avons choisie de restreindre à deux décennies, 19211940, reprenant ainsi la chronologie de nombreux historiens (MEYER, 1973, p.8 et GILLY, 2000, p.340). Cette période est cruciale pour la mise en place du discours idéologique post-révolutionnaire ainsi que pour la délimitation de la symbolique et des idéaux issus du conflit sur lesquels s’appuie le nouveau régime. C’est au cours de ces vingt années que se consolide peu à peu le concept de “mexicanité”, qui s’appuie en particulier sur les photographies de "types mexicains” (PLU JENVRIN, 2005) issues du XIXe siècle. Les images du juan et de la soldadera sont l'expression d'un nouveau type, celui de l'homme et de la femme-soldat, qui se caractérisent par leur courage et leur sacrifice pour le bien de la patrie. Elles participent donc activement à l'affirmation de la "mexicanité". 
Au sein des trois revues illustrées hebdomadaires que nous avons sélectionnées pour retrouver des photographies de la Révolution publiées après 1921, le juan et la soldadera n’apparaissent qu’à une dizaine de reprises. Néanmoins, au vu de leur omniprésence dans le corpus actuel d'images qui circulent sur la guerre civile, l'analyse de ces quelques photographies publiées dans les années vingt et trente nous a semblé indispensable. Les trois revues analysées - Revista de Revistas (créée en 1910), El Universal Ilustrado (fondée en 1917) et Jueves de Excélsior (née en 1923) appartiennent toutes les trois à la catégorie des magazines mondains et frivoles de la capitale mexicaine. Ce sont des hebdomadaires abondamment illustrés, qui s’intéressent avant tout aux faits et gestes de la bonne société de Mexico, mais possèdent néanmoins des rubriques très diverses : théâtre, tauromachie, conseils pour les dames, sciences, actualités internationales et nationales, etc. Les photographies de la Révolution ne font irruption dans ces revues que de façon très ponctuelle après 1921, lors de la commémoration de certains événements précis ou, à la fin des années trente, avec la mode florissante des feuilletons sur la guerre civile.

L'objectif de cette étude est de comprendre comment l'insertion des photographies de juanes et de soldaderas correspond à un retour à l'iconographie des “types mexicains” issus des studios du XIXe siècle tout en contribuant à l'édification des valeurs fondamentales de l'idéologie post-révolutionnaire, qui prennent racine, pour l'essentiel, dans les affrontements de 1910-1920. L'analyse de quelques images précises tente de démontrer le rôle fondamental joué par la photographie de la Révolution dans la consolidation du régime post-révolutionnaire et des idéaux qu’il défendait, dont la "mexicanité".

\section{Le “juan", estampe folklorique d’un héros anonyme}

L’utilisation dans la presse du terme générique juan est moins fréquente que celle de soldadera. La raison en est certainement que, même pour les soldats anonymes, il est plus facile - souvent grâce à leur habillement ou à leur uniforme - de distinguer la faction révolutionnaire à laquelle ils appartiennent que pour les femmes. Ils répondent alors à différentes appellations plus précises et sont qualifiés dans les légendes et les textes de "soldats”, de “révolutionnaires”, de “zapatistes”3, de “constitutionnalistes”, etc. 


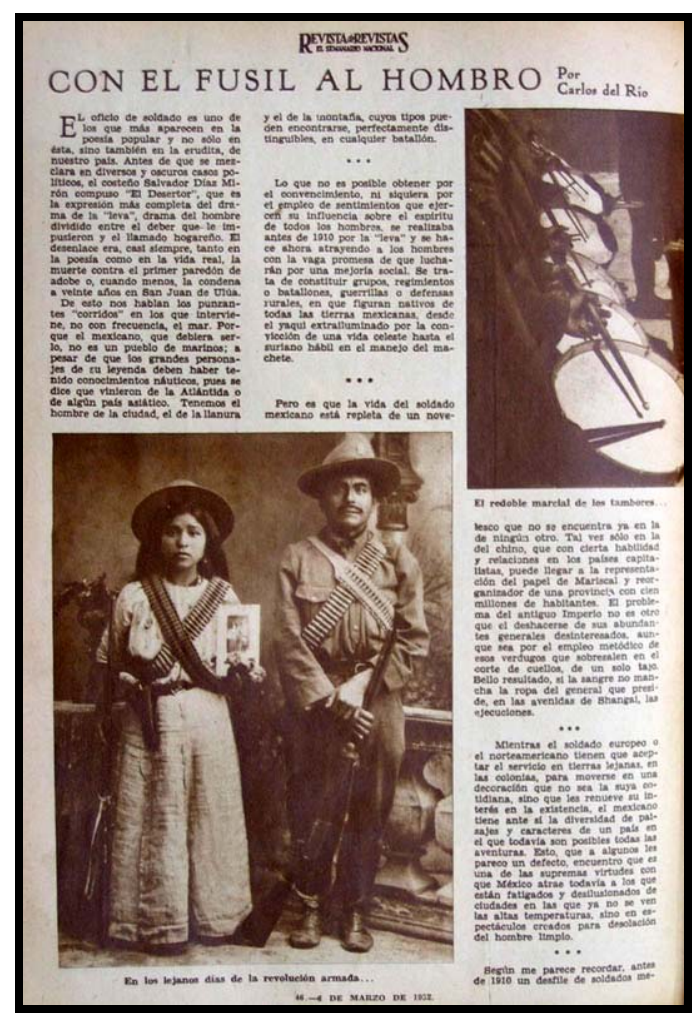

Figure 1 - Revista de Revistas, 6 mars 1932. "Le roulement martial des tambours - Lors des jours lointains de la Révolution armée...” (c) Bibliothèque Miguel Lerdo de Tejada, Mexico, Mexique

Ce couple (figure 1) est très certainement habillé pour la photographie qui semble avoir été prise en studio; la balustrade que l'on aperçoit en bas à droite, ainsi que le vase situé derrière la femme, sont des éléments classiques des décors utilisés dans les ateliers de photographie. Leur habillement est similaire: un chapeau en feutre (Villa en porte un identique sur certaines images), des cartouchières croisées sur le torse et le fusil tenu dans la main droite. L'homme porte des vêtements sombres et des bottes en cuir. La femme est vêtue d'un chemisier et d'une ample jupe en toile de couleur claire. Elle tient dans sa main gauche une photographie dont on peine à distinguer le contenu, mais qui pourrait également être celle d'un couple, peut-être de révolutionnaires.

La double page sur laquelle se trouve ce portrait constitue une sorte d'ode à l'armée mexicaine toujours considérée comme "l'un des plus fidèles aspects du pays”, près de quinze ans après la fin de la guerre civile. ${ }^{5}$ Elle est illustrée par diverses prises du photographe avant-gardiste Agustín Jiménez, révélé dans la presse illustrée au début des années trente. ${ }^{6}$ Les caractéristiques pittoresques et romanesques de la vie de soldat au Mexique sont mises en avant par l'auteur du texte, Carlos del Río: 
Le métier de soldat est l'un de ceux qui apparaissent non seulement le plus souvent dans la poésie populaire, mais également dans la poésie érudite de notre pays. [...] Et c'est que la vie de soldat regorge d'aspects romanesques, que l'on ne trouve plus ailleurs. [...] Les chansons louaient aussi l'abnégation des soldaderas, l'héroïsme des femmes qui se battaient comme leurs hommes et qui traversaient les déserts en portant sur le dos les bagages et les enfants. ${ }^{7}$

Les photographies de juanes ou de solderas sont très fréquemment associées aux $\operatorname{corridos}^{8}$ révolutionnaires qui, la plupart du temps, mettent en scène leurs exploits, leurs sacrifices et la dureté de leur vie dans les trains et sur les champs de bataille. La double page que nous venons de décrire, intitulée “Avec le fusil sur l'épaule”9, ne fait pas exception puisque l'on associe la photographie du couple à la “poésie populaire”. L'authenticité de la photographie de ces jeunes révolutionnaires est donc secondaire étant donné que ce sont les aspects pittoresques et folkloriques du type de vie qu'ils incarnent qui sont censés intéresser le lecteur. Leur présence a pour but de rappeler l’héroïsme du peuple mexicain pendant la Révolution et la spécificité de l'armée mexicaine vis-à-vis des armées étrangères. Par conséquent, la photographie du couple du juan et de la soldadera est ici érigée en symbole de la "mexicanité” et correspond point par point à cette recherche d'une identité nationale propre et nouvelle prônée par les différents gouvernements post-révolutionnaires.

La deuxième fois que Revista de Revistas s’intéresse aux révolutionnaires anonymes, il s'agit plus explicitement encore d'une association entre la musique et les photographies; la page s'intitule “La Révolution dans les strophes des corridos” (figure 2). ${ }^{10}$ Le texte qui accompagne ces trois clichés se penche exclusivement sur les chansons révolutionnaires et en fait l'un des moteurs de l’héroïsme ressenti par le peuple: “Il importe peu qu'[un poète] soit érudit ou populaire, c’est la réaction héroïque qu'il provoque, par la simplicité de sa poésie, chez le peuple, en modelant les esprits, qui est interessante". ${ }^{11}$ Et à propos de l'auteur anonyme du célèbre corrido, "La Adelita”: "Quel poète d'anthologie peut assurer avoir suscité des héroïsmes si nombreux et si purs?”. ${ }^{2}$ 


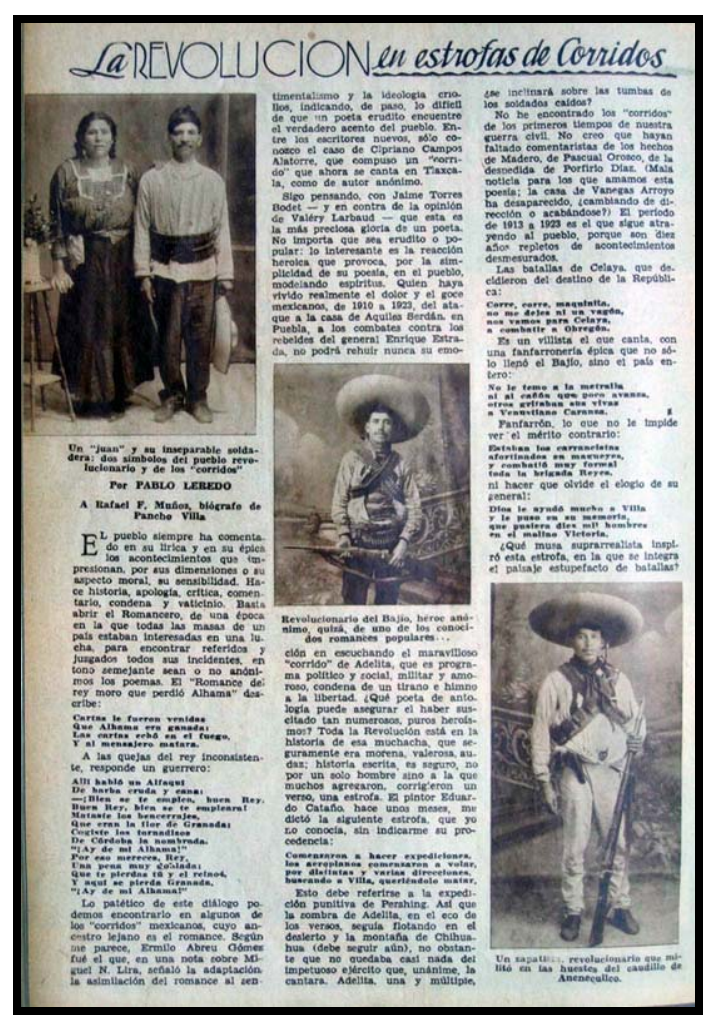

Figure 2 - Revista de Revistas, 20 novembre 1932.”Un juan et son inséparable soldadera: deux symboles du peuple révolutionnaire et des corridos - Révolutionnaire du Bajío, héros anonyme, peut-être de l'une des célèbres romances populaires - Un zapatiste, révolutionnaire qui a milité dans les armées du caudillo d'Anenecuilco ${ }^{13}$ ” (c) Bibliothèque Miguel Lerdo de Tejada, Mexico, Mexique

Alors que le texte n’évoque à aucun moment les images, les mots employés dans les légendes des photographies orientent la lecture de ces images comme des emblèmes de la Révolution: le juan et la soldadera sont qualifiés de "symboles du peuple révolutionnaire” et le soldat que l'on voit sur la deuxième photographie devient un "héros anonyme”, alors qu’il s'agit de façon évidente d’une photographie de composition. ${ }^{14}$ En effet, les deux images du bas ont le même décor en arrière-plan, des escaliers avec une balustrade en fer forgé et des motifs floraux, peut-être sur un rideau ou une toile. Le négatif de l'une des deux photographies a dû être inversé au moment du développement en positif car le décor ne se trouve pas dans le même sens sur les deux clichés. Il s’agit donc de photographies posées, probablement réalisées après la fin du conflit. La constatation de cet artifice photographique enlève toute crédibilité à ce héros de la Révolution, qui n’a peut-être jamais pris part à un combat. La revue ne se soucie pourtant guère de la véracité ou non des légendes qu'elle rédige, cherchant simplement à accorder une représentation à un discours, celui du peuple héroïque. C'est la raison pour laquelle les trois hommes portraiturés sur cette page portent les mêmes attributs: 
des cartouchières, un chapeau à larges bords et un fusil pour les deux hommes seuls. La soldadera n'est nullement associée à la guerre, puisqu'elle ne porte aucune arme. Malgré la ressemblance de leur habillement, la revue fait une distinction entre le révolutionnaire de la région du Bajío ${ }^{15}$ et le révolutionnaire zapatiste. La mise en scène de ce dernier semble assez vraisemblable (pantalon et chemise de toile blanche, cartouchières croisées sur le torse à la façon des partisans de Zapata, et chapeau ample), malgré le port de bottes et non pas de huaraches (MORENO VILLA, 2004, pp.90/91). En revanche, le révolutionnaire du Bajío, hormis le fait qu'il porte un pantalon plus foncé, ne se distingue pas réellement du soldat zapatiste. Force est de constater que le zapatiste l'a emporté sur les autres soldats de la Révolution comme modèle de la représentation du peuple en guerre après 1920. Les muralistes eux-mêmes semblent affectionner le type zapatiste dans leurs peintures. ${ }^{16}$ La raison de cette préférence relève sans doute, et à nouveau, du fait que le zapatiste incarne certaines valeurs de la “mexicanité” telle qu’elle se définit à l’époque, à savoir: l'appartenance au monde rural et à la classe défavorisée. Les trois photographies publiées côte à côte sur cette page démontrent que la presse illustrée ne se soucie guère du réalisme de la représentation des acteurs de la Révolution. Elles annoncent par ailleurs la tendance à l'uniformisation de l'image de la guerre civile, qui s’est accentuée tout au long du XXe siècle, pour laisser place dès les années quarante, et encore aujourd'hui, à une image sans relief et sans diversité du conflit.

Une seule double page (figure 3) rend hommage, non pas au soldat ou au révolutionnaire, mais explicitement au juan. Le titre de cet agencement de cinq images et d'un texte s'intitule d'ailleurs de façon concise: "El "Juan"...”. Le choix des photographies démontre que ce terme s'applique aussi bien aux soldats de l'armée fédérale (voir les trois images de gauche) qu'aux soldats des factions révolutionnaires ne portant ni uniforme, ni képi, mais, au contraire, un chapeau ample (voir les deux images de droite). ${ }^{17}$ Ces photographies ne semblent pas avoir été composées pour les besoins éditoriaux de la revue. L'absence de légendes laisse libre cours à l'interprétation du lecteur sur l'appartenance de chacun de ces hommes à telle ou telle armée. L’observation des uniformes donne néanmoins des indications précieuses. Si le choix des images est plus large que pour les pages précédemment analysées, le texte restreint cependant l'appellation de juan aux seuls indiens: 
[...] il est juste de se souvenir du "Juan”, fait d’héroïsmes obscurs, pétri par les larmes et le sang au cours d'un siècle de conflits internes. Le “Juan soldat”!... L’indien indifférent, stoïque, qui meurt avec une chanson folklorique - fataliste comme sa propre vie - sur des lèvres calcinées par notre soleil. Ce "Juan" victime de tous les tumultes militaires qui suit son chef comme un chien fidèle, sans se soucier de savoir où se trouvent la légalité et la justice. [...] Ce "Juan" enfin mérite que nous tremblions maintenant d'émotion et que nous lui offrions le témoignage graphique de notre profonde sympathie. ${ }^{18}$

Les termes employés par l'auteur de ce texte soulignent à nouveau certaines caractéristiques qu'il semble habituel d'associer au juan: l'héroïsme et le sacrifice. Mais à ces valeurs positives s'ajoutent une description à la teneur assez péjorative: la victimisation du juan et son indifférence face aux causes pour lesquelles il combat. L'accent est d'ailleurs mis davantage sur sa mort que sur ses actions héroïques, alors qu'au contraire, les images montrent des juanes sur les lieux des affrontements ou, du moins, en tenue de combat. L'allusion à la mort du juan est toute entière emprunte de “mexicanité”: cette figure est à nouveau associée à la chanson, précisément définie à cette occasion comme folklorique; elle est également associée au soleil mexicain, élément central de la représentation de l’identité de ce pays.

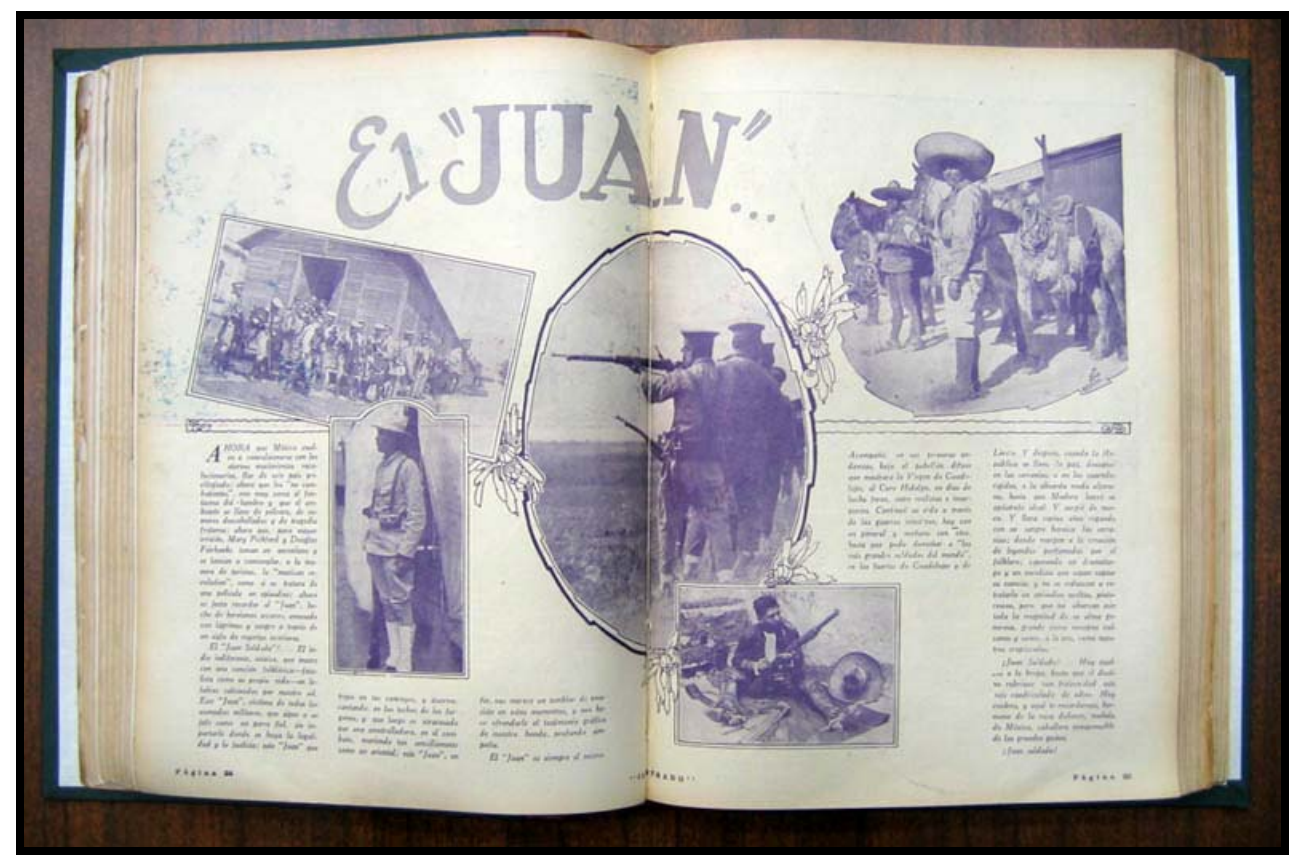

Figure 3 - El Universal Ilustrado, 28 mars 1929. Sans légende (C) Bibliothèque Miguel Lerdo de Tejada, Mexico, Mexique 
Bien que les photographies de cette double page soient plus discrètes dans la représentation folklorique qu'elles donnent du juan, cet iconotexte ${ }^{19}$ (MONTANDON, 1990, p.5) confirme la tendance à la simplification qui sous-tend la commémoration de la vie du soldat révolutionnaire. De façon encore ténue mais discernable, le juan est visuellement caractérisé par son chapeau à larges bords, ses cartouchières, son fusil et son habillement extrêmement simple. Le discours qui lui est associé exalte systématiquement son héroïsme. On observe le même type de schématisation dans la représentation photographique de la soldadera.

\section{La "soldadera", mythe de la femme au combat}

Si des images de soldaderas ne sont publiées qu'à quatre reprises dans les revues illustrées post-révolutionnaires que nous avons sélectionnées, la question de la femme dans la Révolution Mexicaine reste un thème classique de la représentation de la guerre. Elena Poniatowska leur a consacré un livre (PONIATOWSKA, 1999), ainsi que l'Institut National des Études Historiques sur la Révolution Mexicaine (INEHRM, 1993), pour ne citer que deux références. La couverture de la revue Alquimia parue en 2006 à l'occasion du trentième anniversaire du Système National de Photothèques du Mexique est ornée d'une célèbre image de deux soldaderas faisant leurs adieux aux soldats à bord d'un train. ${ }^{20}$ Dans ce même numéro, un article est consacré à l'une des trois photographies les plus répandues de la Révolution actuellement (aux côtés de l'image de Villa et Zapata au Palais National et du portrait en pied de Zapata), celle que l’on appelle aujourd'hui communément “La Adelita”. 21 Miguel Ángel Morales y prétend avoir identifié l'auteur de cette image mythique, qui serait le photographe Jerónimo Hernández; il affirme également que cette femme serait une cuisinière et non pas une prostituée, hypothèse avancée par John Mraz (MRAZ, 2000). Il retrace enfin l'histoire de la publication de cette photographie dans différents ouvrages et recense les rumeurs diverses qui ont couru à son sujet.

La photographie de “La Adelita” est un portrait d'un groupe de huit femmes, juchées sur les marchepieds et les plateformes d'un train, vêtues de jupes amples et

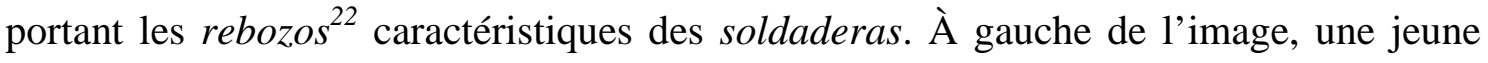
femme se tenant de ses deux mains aux rambardes du train projette son corps en avant et regarde à sa gauche d'un air que l'on pourrait qualifier d'ahuri ou d'effrayé. Quel que soit le sentiment qui habite cette femme, son regard capte celui de l'observateur et 
donne à ce cliché une force visuelle notoire. La photographie de "La Adelita" est d'ailleurs souvent recadrée (elle est coupée en deux dans le sens de la largeur) pour ne mettre en valeur que cette femme. Elle est presque systématiquement réutilisée lorsqu’il s’avère nécessaire d'illustrer des propos sur la Révolution et de nombreuses histoires circulent à son sujet. Cette photographie, qui fait aujourd'hui partie du corpus restreint de la photographie de la Révolution Mexicaine, est un exemple de la "mexicanité” liée à la guerre civile. L'habillement et les accessoires des femmes, ainsi que le train constituant le décor, sont des éléments habituellement repris lors de la représentation iconographique des femmes anonymes de la Révolution, que ce soit à travers des photographies, des dessins ou des peintures. Ne serait-ce qu'en raison de la célébrité de cette seule image, s’arrêter sur les quelques photographies de soldaderas publiées dans les années vingt et trente prend tout son sens.

Avant de se pencher sur les photographies, l'observation d'un dessin réalisé par Fa-Cha ${ }^{23}$ et publié à deux reprises dans Revista de Revistas ${ }^{24}$ est d'un grand intérêt dans ce questionnement sur la représentation visuelle de la soldadera (figure 4).

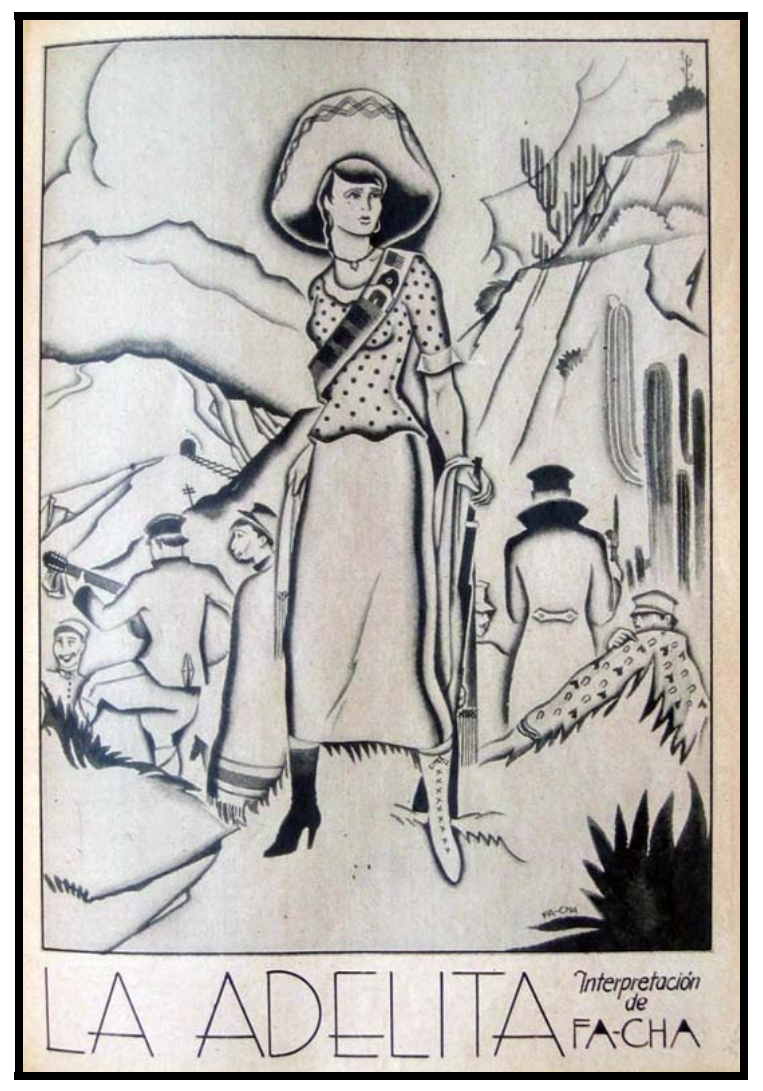

Figure 4 - Revista de Revistas, 20 novembre 1932. “La Adelita” (C) Bibliothèque Miguel Lerdo de Tejada, Mexico, Mexique 
La première fois, ce dessin est publié avec pour seul titre "La Adelita”. La deuxième fois, il vient compléter une double page sur "L'origine de [la chanson] La Adelita $^{25}$. Ces deux emplois différents confirment la double utilisation du terme “Adelita”, à la fois comme nom générique pour la soldadera et comme titre du célèbre corrido. Ce dessin est très riche d'enseignement; on remarque à première vue les trois attributs communément associés au juan: le chapeau à larges bords, le fusil et la cartouchière portée en bandoulière. Un seul attribut diffère, et pour cause, il est exclusivement réservé aux femmes: le rebozo qu'elle porte discrètement derrière sa taille et que l'on aperçoit retombant sur ses poignets. Les vêtements de cette Adelita devraient frapper immédiatement le lecteur: son chemisier à pois cintré, sa jupe étroite et droite qui ne descend qu'au-dessous du genou ainsi que ses bottines à talons et à lacets renvoient davantage à une gravure de mode des années trente qu'à une soldadera évoluant sur les champs de bataille aux alentours de 1914. Le contraste donne à cette Adelita un aspect très folklorique, encore plus éloigné de ce que pourrait être la réalité des photographies. À l'arrière-plan, de nombreux aspects de la “mexicanité” sont réunis: les montagnes, le chemin de fer, la guitare, le sarape ${ }^{26}$ (porté par l'homme à gauche de la soldadera) et les cactus. Cet arrière-plan associe la silhouette féminine à l'identité nationale rappelée par les attributs de la "mexicanité" que nous venons d'énumérer. On remarque à nouveau que la figure de la soldadera s'inscrit dans la représentation de l’image du Mexique recherchée par l’histoire officielle.

Il est plus difficile de rassembler sur une photographie tous les éléments permettant de résumer un idéal, comme le fait ce dessin de Fa-Cha. Néanmoins, les photographies choisies par les revues pour remémorer le rôle des femmes pendant la Révolution évoquent ce même idéal, tout en donnant une représentation plus vraisemblable de ces acteurs spécifiques du conflit. 


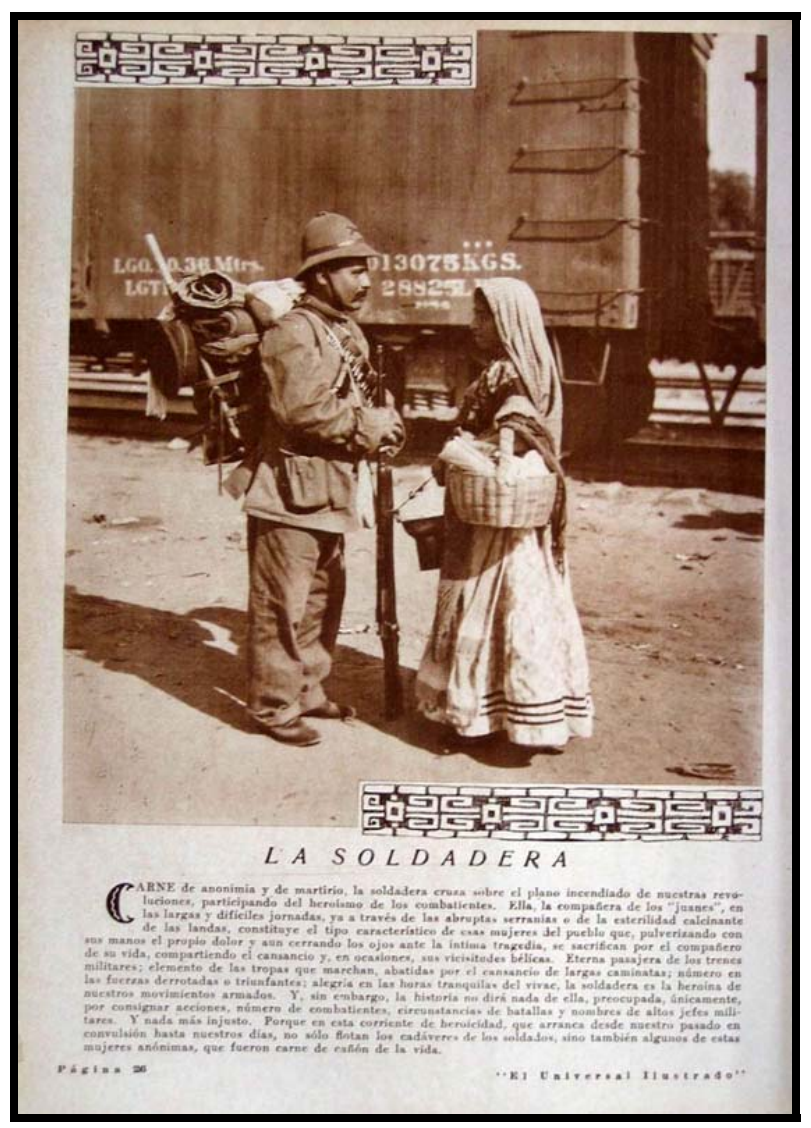

Figure 5 - El Universal Ilustrado, 13 septembre 1928. Sans légende (C) Bibliothèque Miguel Lerdo de Tejada, Mexico, Mexique

Pour honorer la mémoire de la soldadera, El Universal Ilustrado publie une photographie qui a été clairement composée (figure 5). Cette page pourrait d'ailleurs tout aussi bien s’intituler El “juan” que “La soldadera” puisqu'il s'agit du portrait d’un couple de révolutionnaires. Mais la rédaction de la revue a choisi d’insister sur le personnage féminin. L'utilisation de l'article défini dans le titre de la page souligne la volonté de présenter cette femme comme l'incarnation d'un modèle. La soldadera est ici uniquement caractérisée comme femme du soldat et non pas comme femme-soldat. Elle ne porte en effet ni fusil, ni cartouchières, mais un panier et, en bandoulière sur son épaule droite, un comal. ${ }^{27}$ Par conséquent, elle est davantage associée au rôle de cuisinière qu'à celui de combattante. Les vêtements qu'elle porte correspondent bien mieux à ceux des photographies datant de la Révolution que les vêtements dessinés par Fa-Cha pour son Adelita. La jupe est très ample et tombe jusqu'aux pieds, le chemisier est moins près du corps et le rebozo est porté sur la tête, et non pas derrière la taille. Le juan, à qui elle fait face, porte une cartouchière sur le torse et son fusil devant lui; à la 
différence des photographies de juanes observées précédemment, il est vêtu d’un uniforme complet, allant des bottes au chapeau en passant par un sac à dos dans lequel on aperçoit notamment des couvertures. Il est très rare de trouver des images datant de la Révolution sur lesquelles les soldats portent des sacs à dos. Tout autant que l'attitude figée des deux personnages, cette observation nous permet d'affirmer qu'il s'agit d'une photographie de composition. Cette image vise à délimiter clairement le rôle de l'homme et de la femme, celui du soldat et celui de la cuisinière, en les montrant sous un jour favorable: aucun élément ne permet de déceler visuellement les difficultés de la vie de révolutionnaire que le texte dénonce cependant. L’objectif de lecture ${ }^{28}$ de cette page, mieux véhiculé ici par les mots que par l'image, est de souligner le sacrifice des femmes, leur résistance face à l'adversité et la violence à laquelle elles peuvent être confrontées:

Chair anonyme et de martyr, la soldadera traverse le panorama incendiaire de nos révolutions, participant à l’héroïsme des combattants. Elle, la compagne des juanes, lors des longues et difficiles journées, à travers les abruptes montagnes ou la stérilité des landes calcinées, constitue le type caractéristique de ces femmes du peuple qui, pulvérisant avec leurs mains leur propre douleur et en fermant même les yeux face à la tragédie intime, se sacrifient pour le compagnon de leur vie, partageant la fatigue et, parfois, leurs vicissitudes belliqueuses. Éternelle passagère des trains militaires; élément des troupes qui marchent abattues par la fatigue des longues marches; numéro des forces vaincues ou triomphantes, joie des heures tranquilles du bivouac, la soldadera est l'héroïne de nos mouvements armés. Et, cependant, l'histoire ne dira rien sur elle, soucieuse uniquement de consigner des actions, des nombres de combattants, des circonstances de batailles et des noms de grands chefs militaires. Et il n’y a rien de plus injuste. Parce que dans ce courant d'héroïsme, qui commence dans notre passé convulsif pour arriver jusqu'à nos jours, il n’y a pas que les cadavres des soldats qui flottent, mais aussi quelques corps de ces femmes anonymes, qui furent la chair à canon de la vie. ${ }^{29}$

Cette page correspond parfaitement à la définition d'iconotexte que nous avons précédemment donnée; la rédaction d’El Universal Ilustrado a soigneusement associé dans un même espace une photographie, un titre, un court texte et même des frises rappelant des motifs précolombiens. La présence de ces frises vise d'ailleurs vraisemblablement à ancrer la figure de la soldadera dans l'histoire de son pays, en établissant un lien avec le passé précolombien du Mexique. Cet iconotexte délivre une image stéréotypée de la femme révolutionnaire grâce à l’interaction entre le visuel et l'écrit; en effet, si le portrait fige pour longtemps la soldadera dans un "type" photographique déterminé, seul le texte permet de souligner deux caractéristiques de sa 
personnalité: l'héroïsme et le sacrifice. La publication de l'image sans l'univers écrit qui l'entoure n'aurait certainement pas véhiculé le même message. Cette photographie traduit concrètement l'objectif de lecture qui lui est associé: la volonté d'ériger la soldadera en modèle de femme mexicaine courageuse. Par ailleurs, elle laisse entrevoir le processus d'iconisation (NOBLE, 1998, p. 367) qui s'opère pour d'autres photographies de soldaderas, comme pour d'autres sujets faisant partie de l'imagerie révolutionnaire. À partir d'un modèle photographique-type - celui du juan, de la femme-soldat ou du chef révolutionnaire - des images à l'impact visuel fort, comme celle de "La Adelita” ou certains portraits en pied de Zapata vont devenir des imagesicônes. Elles le deviennent en raison de leurs caractéristiques formelles, mais également de leur lien avec un référent de la "mexicanité" issu de la Révolution, par exemple le référent que constitue la soldadera. Le terme iconisation renvoie à cette transformation progressive d'une photographie donnée en image-icône.

Jueves de Excélsior a donné une vision plus large qu'El Universal Ilustrado de ces femmes pendant la guerre (figure 6). Le titre de la double page ne fait d'ailleurs pas explicitement référence à la soldadera. L’objectif recherché est de présenter "Les femmes courageuses de la Révolution”30, tous rôles confondus. La Negra, colonelle zapatiste, ou La Santa, infirmière renommée des champs de bataille, côtoient donc les jeunes filles de bonne société qui ont fondé l’association “Les Filles de la Révolution” à Mexico ou qui se disent sympathisantes de la cause révolutionnaire à Durango. La femme d’Áquiles Serdán ${ }^{31}$, ainsi que Carmen, la sœur de ce dernier, saluées pour leur courage depuis l'assassinat du défenseur de la cause anti-réélectionniste, trouvent également leur place parmi les "femmes courageuses" de la guerre civile. Malgré cette tentative d'élargir le souvenir de la femme pendant la Révolution à toutes sortes de situations différentes, deux photographies sortent du lot grâce à la mise en page: celles de soldaderas anonymes véhiculant à nouveau une image stéréotypée de la femme révolutionnaire. Les deux portraits de couples, détourés, occupent un espace plus important que les autres photographies et sont placés en bas de la page. Dans les deux cas, on observe un geste de tendresse de la femme envers le soldat: celle de gauche embrasse son juan sur la joue, tandis que celle de droite lui tient la main gauche et pose son autre main sur son épaule, tout en fixant l'objectif du photographe. Le soldat de gauche porte un uniforme de l'armée fédérale mais a croisé ses cartouchières sur le torse à la manière des révolutionnaires. Il semble partager le fusil qui se trouve au premier plan avec la soldadera, qui pourrait donc être prise pour une femme-soldat. Le 
couple de droite est uniquement associé à la Révolution grâce aux chapeaux. Avec son panier au bras, la soldadera est ici cantonnée à son rôle de cuisinière. Les deux femmes portent le traditionnel rebozo, tout comme le soldat de droite, ce qui est moins fréquent.

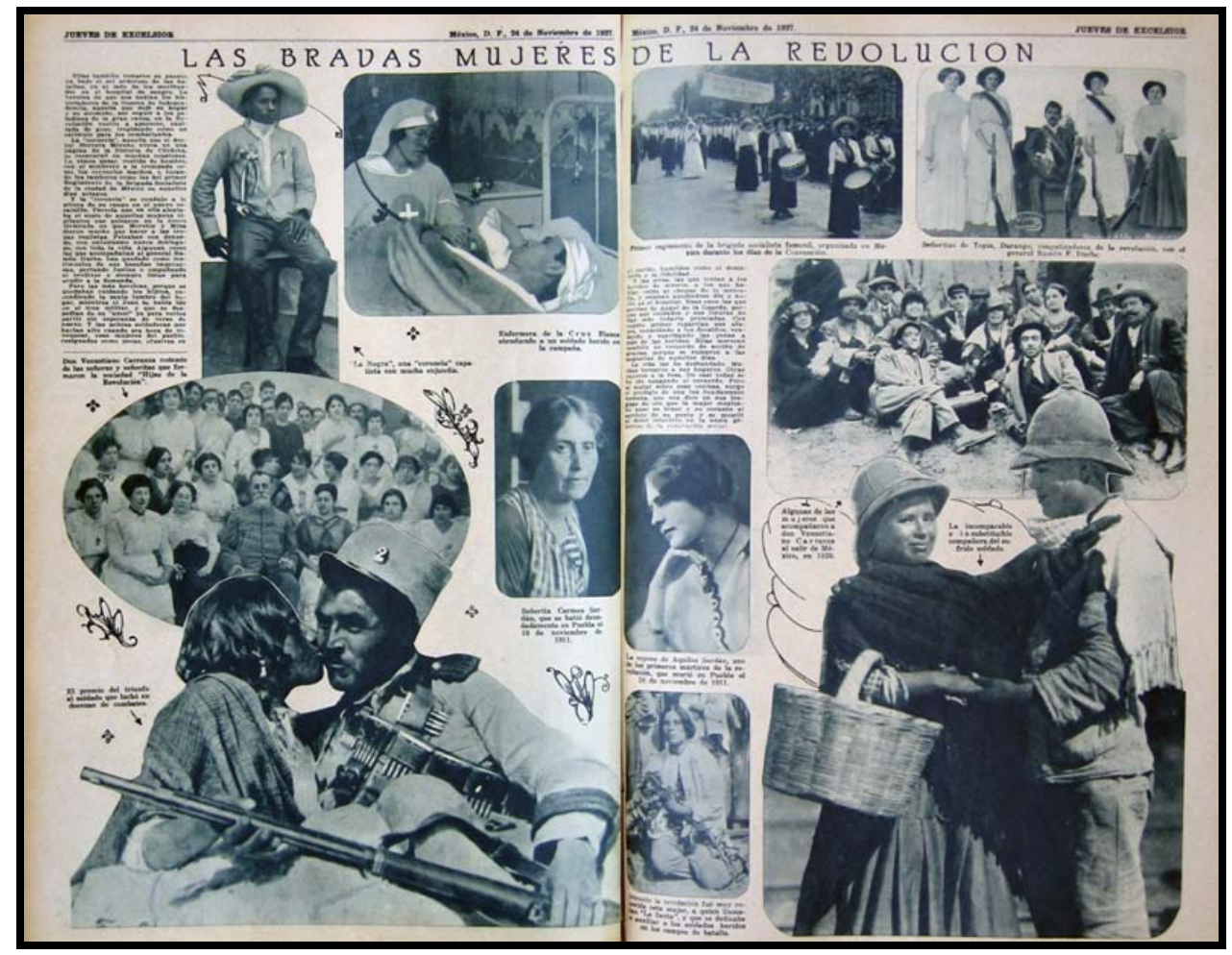

Figure 6. Jueves de Excélsior, 24 novembre 1927. "Page de gauche: La Negra, une colonelle zapatiste avec beaucoup d'envergure - Une infirmière de la Croix Blanche soignant un soldat blessé en campagne - Don Venustiano Carranza entouré des dames et des demoiselles qui ont formé la société "Filles de la Révolution” - Mademoiselle Carmen Serdán, qui s'est battue courageusement à Puebla le 18 novembre 1911 - La récompense du triomphe du soldat qui a lutté dans des dizaines de combats / Page de droite: Premier régiment de la Brigade socialiste féminine, organisée à Mexico pendant la Convention - Demoiselles de Topia, Durango, sympathisantes de la révolution, avec le général Ramón $F$. Iturbe - Certaines des femmes qui ont accompagné Venustiano Carranza lorsqu'il quitta Mexico, en 1920 - La femme d'Áquiles Serdán, l'un des premiers martyrs de la révolution, qui est mort à Puebla le 18 novembre 1911 - L'incomparable et irremplaçable compagne du patient soldat - Pendant la révolution, cette femme fut très connue: on l'appelait $\mathrm{La}$ Santa et elle s'occupait de soigner les soldats blessés sur les champs de bataille” ( Bibliothèque Miguel Lerdo de Tejada, Mexico, Mexique

À la différence des autres femmes présentées sur la page, ces deux-là n’ont pas d’identité individuelle, comme Carmen Serdán, ou collective, comme les membres de la “Brigade socialiste féminine”. Elles ne sont identifiées qu’à travers leur relation avec leur juan: celle de gauche est "la récompense du triomphe du soldat qui a lutté dans des dizaines de combats" tandis que celle de droite est "l'incomparable et irremplaçable compagne du patient soldat”. En dépit d'une volonté manifeste de la part de Jueves de Excélsior d'élargir la commémoration à toutes les femmes de la Révolution, c’est 
encore et toujours le stéréotype de la soldadera anonyme qui se sacrifie pour son juan qui prévaut.

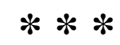

Les revues illustrées, dans les années trente, font encore appel au souvenir des “types mexicains” photographiques du XIXe siècle. La représentation des soldats anonymes, souvent faite à travers des photographies de composition, reprend les règles iconographiques du portrait de studio qui étaient en vigueur pendant le Porfiriat. Il existe donc un paradoxe entre la volonté d'exalter la figure des combattants anonymes, présentés par le discours officiel comme étant à l’origine du triomphe de la Révolution, et, simultanément, leur enfermement dans des “types” à travers la photographie. Malgré l'irruption de la bola ${ }^{32}$ et les bouleversements dans la photographie de presse liés à l'actualité de la guerre civile, le portrait individuel des gens du peuple semble ne pas se défaire aisément des carcans du passé. Et même lors de la commémoration de leur rôle dans la Révolution, le juan et la soldadera sont véritablement traités comme des archétypes de "mexicanité" plutôt que comme des acteurs à part entière du conflit. La schématisation de l'image du juan et de celle de la soldadera au cours de la PostRévolution s’observe également pour les protagonistes les plus reconnus de la guerre, tels que Madero ou Carranza. La réutilisation des photographies révolutionnaires après la fin du conflit participe donc au lissage de la complexité du conflit.

\section{RÉFÉRENCES BIBLIOGRAPHIQUES}

GILLY, Adolfo, "La Révolución Mexicana: ciclos e influencias", in BAILÓN CORRES, Jaime, MARTÍNEZ ASSAD, Carlos, SERRANO ÁLVAREZ, Pablo (Coord.), El Siglo de la Revolución Mexicana, Mexico: INEHRM, 2000, pp.335-341.

Las Mujeres en la Revolución Mexicana. 1884-1920, Mexico: INEHRM, Instituto de Investigaciones Legislativas de la H. Cámara de Diputados, 1993.

MEYER, Jean, La révolution mexicaine, Paris: Calmann-Lévy, 1973.

MONTANDON, Alain (Dir.), Iconotextes, Actes du colloque du 17-19 mars 1988 à l’Université Blaise Pascal de Clermont-Ferrand, Paris: Ophrys, 1990. 
MORENO VILLA, Fernando Ignacio, Los Ejércitos de la Revolución Mexicana, 19101920, Mexico: Senado de la República, 2004.

MRAZ, John, “Historia y mito del Archivo Casasola”, Jornada Semanal, 31 XII 2000.

NOBLE, Andrea, “Zapatistas en Sanborns (1914). Women at the Bar”, History of Photography, vol.22, n4, hiver 1998, pp.366-370.

PLU JENVRIN, Raphaële, “La "Mexicanidad" como soporte para la representación de la institución presidencial en el México post-revolucionario: ¿’hacia qué definición de la nación”, HISTOIRE(S) de l’Amérique latine [En ligne], Volume 1, Numéro 1, 11 octobre 2005.

PONIATOWSKA, Elena, Las soldaderas, Mexico: Era, CONACULTA, INAH, 1999.

GAUTREAU, Marion. The anonymous heroes of the Mexican Revolution on glossy paper : the juan and the soldadera as symbols of "mexicanity". História, São Paulo, v. 26, n. 2, p. 43-60, 2007.

Abstract: Pictures of the mexican civil war from 1910 to 1920, as much as 1920's muralism or "la novela de la Revolución", contributed to the consolidation of post-revolutionary ideology offering the readers of illustrated reviews a soft and stereotyped representation of the conflict. This article basically analyses the pictures of juanes and soldaderas published in three magazines of the Post-Revolution (1910-1920) and demonstrates how these photographies lock up the protagonists of the war into "types" and how they are set up into symbols of "mexicanity", relieving then the simplifying speaches of the post-revolutionnary leaders and erasing the complexity of the conflict that founded Mexico of the XXth century.

Keywords: Juan, Soldadera, Mexican Revolution, Photography, Illustrated Press, Mexicanity»

Artigo recebido em 10/2007. Aprovado em 11/2007.

\section{NOTAS}

\footnotetext{
* Institut d’Études Ibériques et Hispano-américaines de l’Université Paris 4 - Sorbonne ) Doctorat en espagnol de l'Université de Paris 4 - Sorbonne sous la direction de M. le Professeur Sadi Lakhdari (Université Paris 4 - Sorbonne) et de Mme le Professeur Nancy Berthier (Université Paris Est - Marnela-Vallée). marionafmex@yahoo.com

1 Le terme “juan” s’applique à tous les hommes ayant servis dans les différentes armées comme simples soldats au cours de la Révolution Mexicaine.

2 Le terme "soldadera” désigne les femmes de soldats ou les femmes-soldats qui se sont jointes aux différentes factions armées pendant la guerre civile au Mexique.

3 “zapatiste”: sympathisant ou membre des forces d’Emiliano Zapata.
} 
4 “constitutionnaliste”: sympathisant ou membre de l'armée constitutionnaliste, dirigée par Venustiano Carranza.

5 "El ejército es uno de los más fieles aspectos del país”.

6 Voir le numéro de la revue Alquimia intitulé "Agustín Jiménez. La vanguardia”, Alquimia nº11, janvier-avril 2001, $47 \mathrm{p}$.

7 "El oficio de soldado es uno de los que más aparecen en la poesía popular y no sólo en esta, sino también en la erudita, de nuestro país. [...] Pero es que la vida de soldado está repleta de un novelesco que no se encuentra ya en la de ningún otro. [...] Las canciones elogiaban también la abnegación de las soldaderas, el heroísmo de las mujeres que se batían al igual que sus hombres que atravesaban los desiertos llevando en la espalda la impedimenta y los críos”.

8 Corrido: forme de chanson caractéristique du Mexique relatant en plusieurs strophes une tragédie, une aventure, un exploit, etc. Pendant la Révolution, de nombreuses chansons furent créées sur les personnages et les affrontements. Elles connurent une large diffusion pendant et après la guerre civile.

9 "Con el fusil al hombro".

10 "La Revolución en estrofas de corridos".

11 "No importa que [un poeta] sea erudito o popular, lo interesante es la reacción heroica que provoca, por la simplicidad de su poesía, en el pueblo, modelando espíritus”.

12 “¿Qué poeta de antología puede asegurar el haber suscitado tan numerosos, puros heroismos?”.

13 Anenecuilco est le village natal d'Emiliano Zapata. Voir WOMACK, John, Zapata y la Revolución Mexicana, Mexico: Siglo XXI, 1985, pp.2/3.

14 Nous appelons photographie de composition un cliché dont le décor, les costumes et le positionnement des personnages ont été sélectionnés et orchestrés par le photographe. La photographie de composition est souvent considérée comme l’opposé de la photographie instantanée.

15 La région du Bajío se situe au centre du Mexique, autour de la ville de Guanajuato.

16 Voir la reproduction d'un fragment d'une fresque de Diego Rivera sur la couverture d'El Universal Ilustrado le 26 septembre 1935. Ce détail montre Emiliano Zapata revêtant les habits de simple toile blanche propres aux partisans zapatistes.

17 Il semblerait d'ailleurs qu'une photographie de soldat nord-américain se soit glissée dans cette double page. En effet, le portrait du soldat seul, de profil, en bas à gauche de la page, pourrait être un portrait de “l'ennemi”. Le type de bottes qu'il porte n’a jamais été observé pour les uniformes de l'armée fédérale mexicaine.

18 "[...] es justo recordar el "Juan", hecho de heroismos oscuros, amasijado con lágrimas y sangre a través de un siglo de reyertas interiores. El "Juan Soldado"!... El indio indiferente, estoico, que muere con una canción folclórica -fatalista como su propia vida- en los labios calcinados por nuestro sol. Este "Juan" víctima de todas las asonadas militares que sigue a su jefe como un perro fiel, sin importarle donde se halla la legalidad y la justicia. [...] Este "Juan" en fin nos merece un temblor de emoción en estos momentos y nos hace ofrendarle el testimonio gráfico de nuestra honda, profunda simpatia"

19 Nous considérons la page d'une revue illustrée comme un iconotexte car il s'agit d'une architecture mêlant éléments visuels (photographies, dessins, caricatures) et éléments textuels (titre de la page, articles, légendes des images). À l'heure de l'analyse de cette page, ces éléments doivent être pris comme un tout produisant du sens grâce à l'interaction des éléments visuels et textuels entre eux.

20 Alquimia n²7, mai-août 2006, Fototeca Nacional: treinta aniversario.

21 MORALES, Miguel Ángel,’La célebre fotografía de Jerónimo Hernández”, Alquimia n²7, mai-août 2006, pp.68-75.

22 Large foulard porté par les femmes mexicaines, souvent sur la tête.

23 Fa-Cha est la signature du dessinateur de Revista de Revistas, Ángel Zamarripa.

24 Ce dessin a été publié le 20 novembre 1932 ainsi que le 16 juillet 1933 dans Revista de Revistas.

25 "El origen de la Adelita".

26 Sarae: Couverture tissée dont le motif principal sont les rayures.

27 Comal: disque en terre cuite ou en métal que l'on utilise pour faire cuire les galettes de maïs.

28 Ce que nous nommons "objectif de lecture" est la finalité qu'une revue attribue à la publication de certains types de photographies par le biais de leur mode de publication. On peut donc déterminer un objectif de lecture lié à une ou plusieurs photographies en analysant le discours textuel environnant et l'incidence de la mise en page sur l'observation que le lecteur va faire des images.

29 "Carne de anonimia y de martirio, la soldadera cruza sobre el plano incendiado de nuestras revoluciones, participando del heroísmo de los combatientes. Ella, la compañera de los "juanes”, en las largas y difíciles jornadas, ya a través de las abruptas serranías o de la esterilidad calcinante de las landas, constituye el tipo característico de esas mujeres del pueblo que, pulverizando con sus manos el propio 
dolor y aun cerrando los ojos ante la íntima tragedia, se sacrifican por el compañero de su vida, compartiendo el cansancio y, en ocasiones, sus vicisitudes bélicas. Eterna pasajera de los trenes militares; elemento de las tropas que marchan, abatidas por el cansancio de largas caminatas; número en las fuerzas derrotadas o triunfantes; alegría en las horas tranquilas del vivac, la soldadera es la heroína de nuestros movimientos armados. Y, sin embargo, la historia no dirá nada de ella, preocupada, únicamente, por consignar acciones, número de combatientes, circunstancias de batallas y nombres de altos jefes militares. Y nada más injusto. Porque en esta corriente de heroicidad, que arranca desde nuestro pasado en convulsión hasta nuestros días, no sólo flotan los cadáveres de los soldados, sino también algunos de estas mujeres anónimas, que fueron carne de cañón de la vida”.

30 “Las bravas mujeres de la revolución”.

31 Áquiles Serdán, fondateur du Club anti-réélectionniste de Puebla, partisan de Francisco Madero, a été assassiné le 18 novembre 1910, deux jours avant le début officiel de la Révolution. Il est considéré comme le premier martyr de la guerre civile.

32 La bola est un terme qui désigne l'ensemble des hommes qui se sont engagés dans la lutte révolutionnaire au Mexique. De façon plus générale, c'est un terme qui sert à désigner tout type de réunion désordonnée de gens nombreux, lors de divertissements bruyants, de bagarres de rue ou de révolutions. 